\title{
Plantas Medicinais do Cerrado dos Campos Gerais
}

Lucila Kawana Nunes Ferreira ${ }^{1}$, Natalie Alana Pedroso ${ }^{1}$, Juliano Rodrigues Oliveira ${ }^{2} \&$ Lia Maris Orth Ritter Antiqueira ${ }^{1}$

Recebido em 16/06/2020 - Aceito em 25/08/2020

${ }^{1}$ Universidade Tecnológica Federal do Paraná/UTFPR, Brasil.<llucee567@gmail.com, nataliiealana@gmail.com, liaantiqueira@utfpr.edu.br>

${ }^{2}$ Instituto Chico Mendes de Conservação da Biodiversidade/ICMBio, Brasil <juliano.oliveira@icmbio.gov.br>

\begin{abstract}
RESUMO - Este trabalho buscou identificar as espécies nativas do Cerrado com potencial medicinal. A área de estudo compreende a região do Parque Nacional dos Campos Gerais (PNCG), no estado do Paraná. São áreas de ecótono onde o Cerrado faz limite com a Mata Atlântica, na Floresta Ombrófila Mista (Mata de Araucária). As espécies medicinais são conhecidas há muito tempo pelos povos tradicionais. Fazem parte do patrimônio natural, histórico e cultural. O material estudado foi coletado na região do parque conhecida como Buraco do Padre. A identificação das propriedades medicinais foi realizada por literatura, totalizando 43 espécies. As famílias com maior número de espécies medicinais são Myrtaceae e Melastomataceae, com seis e quatro espécies respectivamente, seguidas por Asteraceae e Lauraceae, com 3 espécies cada. O potencial medicinal mais comum entre as espécies é o anti-inflamatório, sendo observado em 20 espécies.
\end{abstract}

Palavras - chave: Buraco do Padre; fitoterápicos; patrimônio natural.

\section{Medicinal Plants of the Campos Gerais Cerrado}

ABSTRACT - This research aimed to identify Cerrado native species with medicinal potential. The study area comprises the Campos Gerais National Park (CGNP) in the state of Paraná, Brazil. These are ecotone areas where the Cerrado meets the Atlantic Forest in the Mixed Ombrophilous Forest (i.e., Araucaria forest). Medicinal species have long been used by traditional folk, as they are part of the natural, historical, and cultural heritage. The studied material was collected in the Buraco do Padre. The identification of medicinal properties was carried out using the available literature, with 43 species in total.The families with the largest number of medicinal species are Myrtaceae and Melastomataceae, with six and four species respectively, followed by Asteraceae and Lauraceae, with 3 species each. The most common medicinal potential among species is anti-inflammatory, being observed in 20 species.

Keywords: Buraco do Padre; phytotherapics; natural heritage.

\section{Plantas Medicinales del Cerrado de Campos Gerais}

RESUMEN - Este trabajo tiene como objetivo identificar especies nativas del Cerrado con potencial medicinal. El área de estudio comprende la región del Parque Nacional Campos Gerais (PNCG), en el Estado de Paraná. Son áreas de frontera ecotónica o cerrada con Mata Atlántica, en la Selva Mixta (Bosque de Araucaria). Se sabe que las especies medicinales tienen el pelo largo tradicional. Forman parte del patrimonio natural, histórico y cultural. El material estudiado fue recolectado en la región del parque conocido como Buraco do Padre. La identificación de propiedades medicinales se realizó mediante la literatura, totalizando 43 especies. Las familias con mayor número de especies medicinales son Myrtaceae y Melastomataceae, con seis y cuatro especies respectivamente, seguidas de Asteraceae y Lauraceae, con 3 especies cada una. El potencial medicinal es más común entre especies y antiinflamatorio, observándose en 20 especies.

Palabras clave: Buraco do Padre; fitoterapia; patrimônio natural. 


\section{Introdução}

O bioma Cerrado possui como característica peculiar a vegetação rasteira, repleta de gramíneas $e$ arbustos, com árvores baixas de troncos retorcidos, com folhas rígidas. Sua maior área de ocorrência é a região do Brasil central, mas se expande ao sul até a região dos Campos Gerais, onde inicia a ocorrência da Mata Atlântica (Ritter et al., 2010).

Por se tratar de área de ecótono, incluindo a presença de elevado número de espécies raras, endêmicas e ameaçadas de extinção, há necessidade de estudos relacionados à biodiversidade do Cerrado dos Campos Gerais. Há que se considerar também a forte pressão da expansão do agronegócio, que cria condição de elevada vulnerabilidade desses remanescentes, que muitas vezes estão em pequenas "ilhas" ou "encraves" em meio às áreas agrícolas (Antiqueira \& Moro, 2020).

Dentre as espécies vegetais do Cerrado brasileiro, muitas são utilizadas por comunidades tradicionais de cada região, devido às importantes propriedades medicinais que possuem (Oliveira, 2011). Além disso, muitas possuem potencial alimentício "não convencional", dadas suas características nutricionais, demonstrando que, além de sua importância ecológica, o Cerrado está entrelaçado com o patrimônio cultural e histórico dos povos.

As espécies medicinais do Cerrado brasileiro já possuem alguns estudos científicos, deixando de ser parte somente da medicina tradicional de povos primitivos e passando a serem consideradas pela ciência como possibilidades de avanço em tratamentos de saúde para cura e alívio de enfermidades. No entanto, apesar das pesquisas $e$ avanços no conhecimento farmacológico, devese levar em consideração a importância do papel que as tradições familiares possuem até hoje, pois delas surgiram a observação, a experimentação e o aprendizado a partir da interação com a natureza, utilizando-a para busca da saúde e religião (Ramos et al., 2019).

Nesta pesquisa foi realizado um levantamento etnobotânico de espécies medicinais nativas do Cerrado dos Campos Gerais, a partir de coletas em campo na área do Buraco do Padre e de informações da literatura.

\section{Importância de plantas medicinais ao longo da história}

Segundo Monteiro \& Brandelli (2017), plantas medicinais são espécies utilizadas para fins terapêuticos, cultivadas ou não, frescas ou não (quando são utilizados processos de secagem para armazenamento). O costume de utilizar plantas para tratamentos medicinais faz parte de um dos primeiros esforços do ser humano para compreender a natureza, buscando amenizar $e$ curar males.

Os registros de uso de plantas medicinais vêm de quase três mil anos antes de Cristo (a.C.), nas regiões da Mesopotâmia e China. Registros em papiros do Antigo Egito evidenciam que, a partir de dois mil a.C., as doenças já eram consideradas causas naturais por diversos médicos, que utilizavam as plantas medicinais como base para os tratamentos.

Por volta de 2.300 a.C, os egípcios, hebreus e assírios criaram classes de medicamentos com base na aplicação de espécies conhecidas. $\mathrm{Na}$ Índia, os princípios da Ciência da Vida (Ayurveda) também incluem fitoterapia e são utilizados desde 1.500 a.C.

No início da Era Cristã, o médico e militar grego Pedanius Dioscórides catalogou e ilustrou cerca de 600 plantas medicinais, as quais organizou no livro De Materia Medica, utilizado como principal referência no Ocidente até o período do Renascimento (Monteiro \& Brandelli, 2017).

$\mathrm{Na}$ Idade Moderna, um dos principais defensores das plantas medicinais foi Paracelso (Philippus Aureolus Theophrastus Bombastus von Hohenheim - 1493/1541), que designou tratamentos específicos para cada doença conhecida de sua época e região, além de buscar novos medicamentos com base nos produtos naturais disponíveis. Mas foi apenas a partir do século XIX que ocorreram os maiores avanços no campo de ervas medicinais, com contribuição dos progressos científicos que estavam ocorrendo (Monteiro \& Brandelli, 2017).

No Brasil, a influência dos jesuítas, indígenas e africanos (escravos e livres), contribuiu para o conhecimento e popularidade das plantas medicinais (Almeida, 2011). A cultura de uso se consolidou, de forma que é utilizada até hoje, tanto por indivíduos que não possuem condições 
financeiras para custear tratamento médico, como também por tradição familiar, motivos religiosos (fé) ou mesmo para evitar os efeitos colaterais causados pela ingestão de medicamentos sintetizados (Brasil, 2019).

$\mathrm{O}$ uso de fitoterápicos com finalidade profilática, curativa, paliativa ou com fins de diagnóstico passou a ser oficialmente reconhecido pela Organização Mundial da Saúde (OMS) em 1978, quando foi recomendada a difusão mundial dos conhecimentos necessários para o seu uso (Brasil, 2006). Importante esclarecer que os fitoterápicos são elaborados a partir de plantas medicinais, com uma formulação específica. A definição para planta medicinal dada pela OMS é "todo e qualquer vegetal que possui, em um ou mais órgãos, substâncias que podem ser utilizadas com fins terapêuticos ou que sejam precursores de fármacos semi-sintéticos" (OMS, 1998).

Joy (1998) divide as plantas medicinais em quatro grandes grupos, de acordo com sua função e os sintomas que o indivíduo apresenta: (i) doenças do aparelho digestivo; (ii) problemas respiratórios; (iii) verminose; e (iv) cicatrização. No entanto, explica que há outras classificações, de acordo com as partes utilizadas da espécie vegetal ou habitat em que se encontram.

O Brasil, detentor da maior biodiversidade mundial, possui centenas de espécies com potencial medicinal. No entanto, pesquisas voltadas para a etnobotânica (que utiliza os campos da botânica $e$ da antropologia para compreender a interação dos humanos com as plantas) ainda são consideradas desafiadoras, devido à contínua destruição de habitat que leva à extinção das espécies, o que dificulta a realização de estudos antes que as espécies sejam perdidas, principalmente por meio do desmatamento (Amoroso, 2002).

O Cerrado é abundante em espécies medicinais, muitas delas popularmente utilizadas, devido aos conhecimentos de povos tradicionais. Das mais de dez mil espécies vegetais nativas da savana brasileira, pelo menos duzentas delas são utilizadas na medicina popular (Pereira et al., 2007), assim como na alimentação humana, incluindo as denominadas plantas alimentícias não convencionais (PANC) (Avidos \& Ferreira, 2000).

Entre os habitantes das áreas de Cerrado, residem quilombolas, indígenas, pescadores, artesãos, entre outros, que são denominados povos tradicionais, além de serem os principais consumidores das plantas medicinais do bioma, tanto por falta de recursos financeiros como por tradição familiar, esta última sendo resultado da interação entre os humanos e a natureza. A OMS reconhece que a medicina popular se embasa não apenas na observação e experimentação, mas também nas crenças e no contexto cultural onde uma sociedade se encontra (OMS, 1998).

\section{Material e Métodos}

A região dos Campos Gerais, no estado do Paraná, possui fisionomia de campos limpos e cerrados localizados na borda do Segundo Planalto Paranaense (Maack, 1948). Apesar de ser constituída por uma vegetação relictual, é naturalmente esperada a expansão de florestas estacionais, florestas com araucária e ecótonos entre essas áreas, devido ao aumento natural de temperatura e umidade que ocorre gradativamente nos últimos milhares de anos (Melo et al., 2014).

A criação do Parque Nacional dos Campos Gerais (PNCG) foi proposta em 2005 pelo Ministério do Meio Ambiente (MMA) e homologada por decreto no ano de 2006, visando a "preservação dos últimos remanescentes da floresta de araucárias e a criação de corredores ecológicos com o objetivo de garantir a interligação e manutenção do fluxo gênico entre os principais fragmentos" (Melo et al., 2014). Nessas áreas também se encontram remanescentes de Cerrado, representando o limite austral de ocorrência do bioma no Brasil (Ritter et al., 2010).

A paisagem do PNCG "representa uma típica associação entre a floresta de araucária e campos nativos de grande potencial cênico", sob os afloramentos de rochas na Escarpa Devoniana. Regiões geomorfológicas singulares também fazem parte do parque, compondo, inclusive, outras unidades de conservação municipais $e$ estaduais, como o Buraco do Padre, a Cachoeira Mariquinha, as Furnas Gêmeas e o Canyon do Rio São Jorge (Melo et al., 2014).

As coletas foram realizadas na área do Buraco do Padre (Figura 1), que é uma das mais visitadas devido à beleza da furna, que possui em seu interior uma cascata de, aproximadamente, 30 metros de altura, formada pelo rio Quebra Perna, ao qual rios subterrâneos e túneis se associam, sendo um importante aquífero estrutural (Melo et al., 2005). 


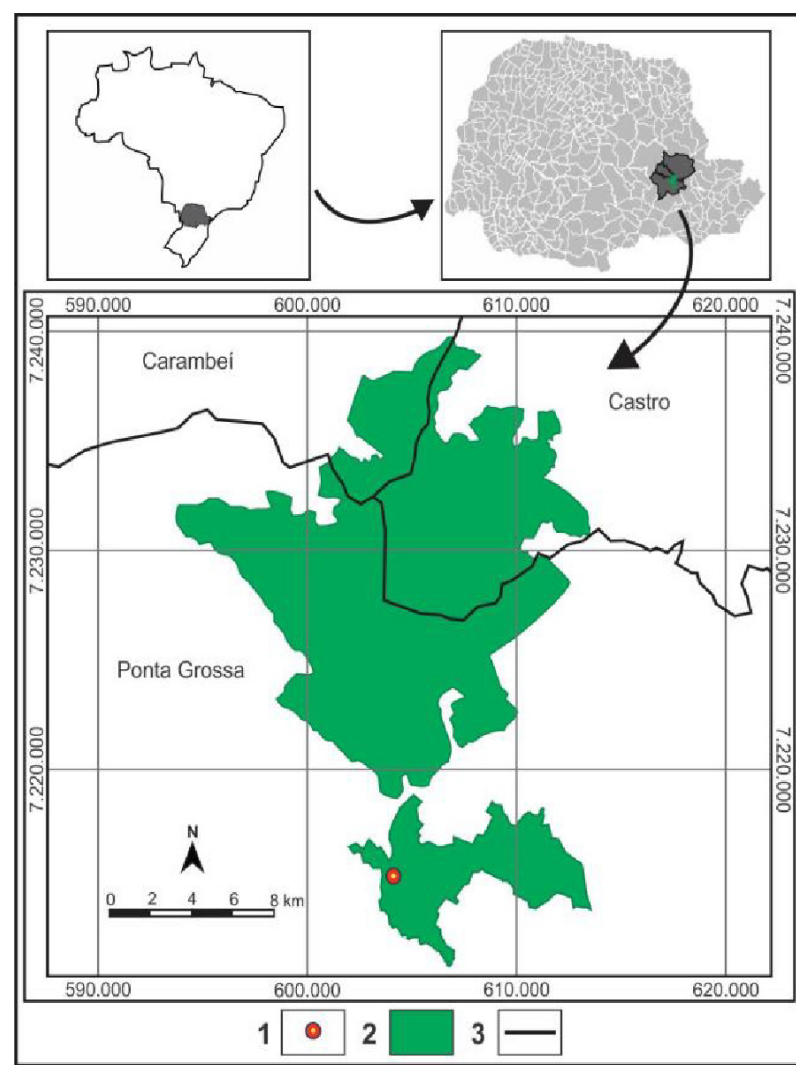

Figura 1- Localização do Parque Nacional dos Campos Gerais (em verde), no estado do Paraná. $\mathrm{O}$ ponto vermelho indica a área de coleta (Buraco do Padre). Fonte: Massuqueto et al. (2019).
A metodologia utilizada nas expedições foi a de Caminhamento, proposta por Filgueiras (1994), percorrendo toda a trilha que leva do Centro de Visitantes até o topo da furna, local onde se concentram os remanescentes de Cerrado (2511'4.25"S e 4958'20.94"O).

O material coletado foi herborizado seguindo as recomendações de Fidalgo e Bononi (1989) e armazenado no Herbário da Universidade Tecnológica Federal do Paraná em Ponta Grossa. A identificação foi realizada com base em literatura específica e em comparação com os exemplares acervados no Herbário da Universidade Estadual de Ponta Grossa.

Os dados sobre potencial medicinal das espécies nativas do Cerrado e os usos recomendados foram consultados em literatura especializada.

\section{Resultados e Discussão}

Foi possível identificar 43 espécies do Cerrado dos Campos Gerais com propriedades medicinais. Essas espécies pertencem a 26 famílias botânicas. As famílias com maior número de espécies medicinais são Myrtaceae e Melastomataceae, com 6 (seis) e 4 (quatro) espécies, respectivamente. A terceira maior ocorrência de espécies é nas famílias Asteraceae e Lauraceae, com 3 (três) espécies cada (Tabela 1).

Tabela 1 - Famílias e espécies do Cerrado dos Campos Gerais com indicação de uso medicinal.

\begin{tabular}{|c|c|}
\hline FAMÍLIA/NOME CIENTÍFICO & INDICAÇÃO E FORMA DE USO SEGUNDO LITERATURA \\
\hline \multicolumn{2}{|l|}{ AMARANTHACEAE } \\
\hline Pfaffia tuberosa (Spreng.) Hicken & $\begin{array}{l}\text { Tônico para estresse, infertilidade, afrodisíaco e estimulante. Chá feito a partir das } \\
\text { raízes. }\end{array}$ \\
\hline \multicolumn{2}{|l|}{ ANNONACEAE } \\
\hline Guatteria australis A.St.-Hil. & $\begin{array}{l}\text { Antibacteriana, auxilia na melhora do sistema imunológico. Chá feito a partir da } \\
\text { casca da fruta. }\end{array}$ \\
\hline \multicolumn{2}{|l|}{ AQUIFOLIACEAE } \\
\hline Ilex paraguariensis St.Hill. & $\begin{array}{l}\text { Possui propriedades diuréticas, estimulantes e estomacais. Infusão feita a partir das } \\
\text { folhas. }\end{array}$ \\
\hline \multicolumn{2}{|l|}{ ASTERACEAE } \\
\hline $\begin{array}{l}\text { Moquiniastrum polymorphum (Less.) } \\
\text { G. Sancho. }\end{array}$ & $\begin{array}{l}\text { Tratar afecções bronco-pulmonares e reumatismo ósseo. Chá obtido a partir das } \\
\text { folhas e da casca do caule. }\end{array}$ \\
\hline Senecio brasiliensis (Spreng.) Less. & $\begin{array}{l}\text { Anti-inflamatória, antibacteriana, antiviral, analgésica, antitumoral e antioxidante, } \\
\text { utilizada, principalmente, para tratar dor de estômago e úlcera gástrica, queimadu- } \\
\text { ras e alergias. Infusão feita a partir das folhas. }\end{array}$ \\
\hline \multicolumn{2}{|l|}{ ASTERACEAE } \\
\hline $\begin{array}{l}\text { Baccharis oblongifolia (Ruiz \& Pav.) } \\
\text { Pers. }\end{array}$ & $\begin{array}{l}\text { Hepatoprotetora, utilizada, principalmente, para tratar desordens renais e diabetes. } \\
\text { Chá feito a partir das folhas. }\end{array}$ \\
\hline
\end{tabular}




\section{FAMÍLIA/NOME CIENTÍFICO}

\section{BIGNONIACEAE}

Pyrostegia venusta (Ker Gawl) Miers

Jacaranda oxyphylla Cham.

\section{BROMELIACEAE}

Tillandsia tenuifolia L.

\section{CELASTRACEAE}

Plenckia populnea Reissek.

Eriosema glabrum Mart. ex Benth.

\section{COMMELINACEAE}

Commelina erecta $\mathrm{L}$.

\section{CONVOLVULACEAE}

Cuscuta racemosa Mart.

\section{CUCURBITACEAE}

Cayaponia espelina (Manso) Cogn.

Gaylussacia brasiliensis (Spreng.)

Meisn.

\section{ERYTHROXYLACEAE}

Erythroxylum deciduum A.St.-Hil.

EUPHORBIACEAE

Croton antisyphiliticus Mart.

\section{FABACEAE}

Dalbergia frutescens (Vell.) Britton

Periandra mediterranea (Vell.) Taub..

\section{IRIDACEAE}

Sisyrinchium vaginatum Sprengel.

\section{LAMIACEAE}

Aegiphila verticillata Vell.

\section{LAURACEAE}

Ocotea bicolor (Vattimo-Gil).

Nectandra grandiflora Nees.

Nectandra grandiflora Nees \& Mart. ex Nees.

\section{MELASTOMATACEAE}

Leandra lacunosa Cogn. Miconia sellowianaNaudin.

\section{INDICAÇÃO E FORMA DE USO SEGUNDO LITERATURA}

Anti-inflamatória e antioxidante, auxilia no tratamento de bronquite e asma. Infusão a partir das flores ou ramos.

Indicada no tratamento de doenças venéreas e afecções cutâneas. Chá feito a partir das folhas.

Diurética, auxilia no tratamento contra processos inflamatórios. Chá feito a partir dos ramos.

Antidisentérico, auxilia também no tratamento de alergias e ferimentos. Chá feito a partir dos ramos e folhas.

Laxante. Pequenas doses de infusão a partir das folhas.

Indicada para tratamentos contra inflamação, especialmente na região dos olhos Infusão feita a partir das folhas.

Anti-inflamatório, diurético, auxilia no tratamento de ferimentos e distúrbios estomacais. Chá feito a partir dos ramos.

Antidiarreica, antirreumática, ameniza dores e manchas no rosto, anti-inflamatório, indicado para tratar feridas e úlceras. Chá feito a partir das folhas ou raízes.

Possui atividade antitumoral e anti-inflamatória, indicada, principalmente, para o tratamento de feridas, além de ajudar a reduzir o colesterol e prevenir infecção urinária. Indicada tanto para ação local quanto oral.

Antioxidante, anticancerígeno e anti-inflamatório. Infusão feita a partir das folhas.

Auxilia no combate contra infecções no sistema reprodutor, tanto masculino quanto feminino. Chá feito a partir das raízes.

Antisifilítica, ajuda a combater diarreia e blenorragia. Infusão feita a partir das folhas.

Anti-inflamatória, auxilia no tratamento de problemas respiratórios. Chá feito a partir das raízes.

Utilizada para controle de natalidade e indução de aborto. Chá feito a partir dos ramos da planta.

Utilizada, principalmente, no tratamento de dengue e picadas de cobras venenosas. Chá feito a partir das folhas.

Anti-inflamatória, antibacteriana e antifúngica. Chá feito a partir da casca.

Diurética, antirreumática, diurética e digestiva. Chá feito a partir da casca.

Antitumoral e anti-inflamatória. Chá feito a partir da casca.

Adstringente. Chá feito a partir das folhas.

Calmante, cicatrizante e anti-inflamatório. Chá feito a partir das folhas. 


\section{FAMÍLIA/NOME CIENTÍFICO}

Miconia hyemalis A.St.-Hil. \& Naudin.

Miconia cinerascens Miq. MENISPERMACEAE

Cissampelos ovalifolia DC.

\section{MORACEAE}

Sorocea bonplandii (Baill.)

W.C.Burger et al..

MYRTACEAE

Eugenia pitanga (O.Berg) Nied..

Eugenia punicifolia (Kunth) DC

Blepharocalyx salicifolius (Kunth) O.

Berg.

Campomanesia adamantium

(Cambess.) O. Berg.

Psidium australe Cambess.

Paramyrciaria delicatula (A.DC.)

Kausel.

\section{ORCHIDACEAE}

Epidendrum secundum Jacq.

\section{PIPERACEAE}

Piper gaudichaudianum Kunth.

RUBIACEAE

Palicourea rigida Kunth.

Psychotria carthagenensis Jacq.

\section{SAPINDACEAE}

Matayba elaeagnoides Radlk..

\section{SYMPLOCACEAE}

Symplocos uniflora (Pohl.) Benth.

Adstringente, auxilia na digestão e no tratamento de febres tropicais. Chá feito a partir das raízes da planta ou da casca da planta. A raiz também pode ser ingerida crua.

\section{VERBENACEAE}

Lantana camara L.

Anti-hemorrágico, diurético, antirreumático, anticonvulsivo e expectorante. Chá feito a partir das folhas ou raízes da planta.

\section{INDICAÇÃO E FORMA DE USO SEGUNDO LITERATURA}

Calmante, auxilia na regulação dos batimentos cardíacos, anti-inflamatória. Chá feito a partir das folhas.

Anti-inflamatória, cicatrizante, calmante. Chá feito a partir das folhas.

Adstringente, antirreumático, anti-inflamatório, antídoto contra veneno de cobras, diurético, tônico. Chá feito a partir das raízes.

Indicada para problemas digestivos. Chá feito a partir das folhas ou banho com a infusão de pedaços da casca.

Antirreumática, antidiarreica, febrífuga e estimulante. Chá feito a partir das folhas. Considerada uma insulina vegetal, é indicada para o tratamento de diabetes. Chá feito a partir das folhas.

Antitumoral, anti-inflamatória, antifúngica e antioxidante. Chá feito a partir das folhas.

Anti-inflamatória, antisséptica e antidiarreica. Chá feito a partir das folhas ou consumo de frutos frescos.

Antidiarreica, diurética e antioxidante. Chá obtido a partir das folhas ou da casca da fruta.

Propriedades antioxidantes. Chá feito a partir das folhas.

Antitumoral, anti-inflamatória, antirreumática e antibacteriana. Chá obtido a partir das folhas ou pétalas da planta.

Anti-inflamatória e indicada para distúrbios hepáticos. Mastigar as raízes ou folhas frescas da planta, ou infusão feita a partir das folhas.

Antimicrobiana, utilizadas em infecções do trato urinário. Chá feito a partir das folhas.

Antioxidante, tradicionalmente utilizada em rituais religiosos como constituinte de bebida alucinógena. Chá feito a partir das folhas.

Antirreumático, antitérmico, anti-inflamatório, digestivo, indicado para azias e problemas no fígado e dores no coração. Chá feito a partir da casca ou raiz da planta.

Fonte: Theoduloz et al. (1988); Adebajo et al. (1989); Bolzani et al. (1992); Leitão et al. (1996); Moreira et al. (2001); Mendonça et al. (2005); Almeida et al. (2015); Almeida (2011); Brasil (2006); Pereira et al. (2007); Lima (2010); Oliveira (2011); Oliveira et al. (2011); Vieira (2011); Nascimento et al. (2013); Rocha et al. (2015); Ramos et al. (2019). 
Com base nos dados obtidos, é possível verificar que as espécies medicinais encontradas no Cerrado dos Campos Gerais apresentam usos bastante distintos verificados em literatura especializada, embora seja possível notar que determinadas espécies possuem algumas aplicações em comum, como é o caso do potencial anti-inflamatório.

Apesar de as espécies citadas estarem vinculadas ao uso na medicina tradicional, elas não estão limitadas apenas ao uso popular. Muitas delas são constantemente estudadas de forma científica e sistematizada, devido ao seu grande potencial farmacológico. E há espécies que já são exploradas comercialmente, com cultivo em grande escala (ex: Ilex paraguariensis) ou pequena escala (ex: Piper gaudichaudianum), e comércio se realiza em feiras livres e lojas de produtos naturais (Miranda \& Urban, 1998; Guimarães \& Monteiro, 2006).

A espécie Erythroxylum deciduum, árvore de grande porte, popularmente conhecida como "cocão", destaca-se por suas propriedades antiinflamatórias, antioxidantes e antitumorais. Apesar de seus frutos apresentarem efeitos tóxicos para ovinos, suas folhas possuem atividade anticancerígena com um grande potencial citotóxico em linhagens de leucemia, estas cultivadas in vitro (Nascimento et al., 2013).

A espécie Pyrostegia venusta, trepadeira conhecida como "cipó-de-são-joão", possui propriedades antimicrobianas, antioxidantes $e$ anti-inflamatórias. Apresenta efeitos inibitórios sobre diversos microrganismos, além de reduzir células inflamatórias, potencializando assim o processo de cicatrização de ferimentos. Além disso, um estudo realizado com o extrato aquoso dessa planta em modelo animal apresentou a redução dos principais sintomas da asma, além de aumentar a capacidade antioxidante, podendo vir a tornar-se uma alternativa aos medicamentos sintetizados, os quais, por vezes, apresentam efeitos colaterais que podem prejudicar o indivíduo (Balestra, 2015).

Aegiphila verticillata, comumente chamada de "fruto-de-papagaio", é bastante conhecida por sua ação contra doenças transmitidas pelo mosquito Aedes aegypti (Mendonça et al., 2005), além de auxiliar como antídoto no tratamento de picada de cobras venenosas (Leitão et al., 1996).
A espécie de grande porte Eugenia pyriformis, conhecida como "uvaia", é capaz de inibir a enzima xantina-oxidase, motivo pelo qual é utilizada no tratamento de gota (Theodoluz et al., 1988).

O fruto de Campomanesia adamantium, arbusto que pode chegar até dois metros de altura, conhecido popularmente como "guavira" (Vieira et al., 2011), atua na recomposição da flora intestinal (Lorenzi et al., 2006).

Um estudo da composição química de um óleo essencial das folhas em estado fresco de Eugenia pitanga (O. Berg) Nied., a "pitanga-docerrado", demonstrou atividade antiparasitária contra o parasita Leishmania amazonensis, responsável pela leishmaniose, uma infecção parasitária (Adebajo et al., 1989).

Piper gaudichaudianum, arbusto de pequeno porte conhecido como "pariparoba" possui atividades analgésicas que se comparam a analgésicos anti-inflamatórios não-esteroidais, além de apresentar atividade antiulcerogênica (Moreira et al., 2001).

Palicourea rigida, popularmente conhecida como "gritadeira", é bastante utilizada para tratar inflamações no trato urinário, especialmente nas regióes do Cerrado brasileiro, onde é mais conhecida (Bolzani et al., 1992).

Além do potencial na medicina tradicional e das pesquisas científicas, as plantas medicinais também possuem grande valor religioso dentro de determinadas culturas, como é o caso de Psychotria carthagenensis, conhecida popularmente como "carne-de-vaca": um arbusto de dois a três metros de altura, originário do Cerrado brasileiro, e distribuído pelo continente sul-americano. Essa espécie serve como parte integrante do preparo da bebida alucinógena "santo-daime", utilizada por povos primitivos da Amazônia, tanto para ritos religiosos, como também para propósitos medicinais e sociais (Lima, 2011).

Não foram localizados registros de estudos voltados para plantas medicinais do Cerrado nos Campos Gerais, apenas estudos desenvolvidos em outras regióes do Brasil, em áreas nucleares do bioma (Pereira et al., 2007). Porém, conforme apontam Antiqueira \& Moro (2020), há grande expressividade na diversidade de espécies do Cerrado, sendo que, em algumas regiões, o endemismo pode chegar a 70\%. Essas informações 
corroboram a necessidade de estudos voltados para a peculiaridade das formações savânicas do PNCG, embora haja espécies identificadas que possuem ocorrência em outras formações campestres e mesmo florestais.

As informações de potencial medicinal obtidas em literatura evidenciam a importância que a biodiversidade do Cerrado apresenta sob diferentes aspectos (natural, histórico e cultural) e a importância da preservação dos recursos naturais.

\section{Conclusões}

Este é um estudo preliminar que buscou contribuir nos aspectos básicos de sensibilização para a conservação da natureza. Na sequência será pesquisada mais a fundo a histórica interação dos povos tradicionais com a natureza, que lhes permite a extração dos recursos necessários para sobrevivência sem negligenciar a preservação da biodiversidade.

Estão previstas também a identificação de mais espécies com potencial medicinal $e$ alimentício não convencional, para as quais serão levantadas possibilidades de uso. Pretende-se, assim, fornecer subsídios que contribuam para a proteção da biodiversidade, por meio do incentivo às práticas sustentáveis de uso dessas espécies, além de resgatar a cultura a elas atrelada.

\section{Agradecimentos}

Ao ICMBio, por autorizar e apoiar a realização das atividades deste projeto na unidade de conservação. À Fundação Araucária, por concessão de bolsa de Iniciação Científica. Ao grupo de pesquisa CONEA - Conservação da Natureza e Educação Ambiental da UTFPR Ponta Grossa, pelo apoio nas atividades de campo e de laboratório.

\section{Referências}

Adebajo AC, Oloki KJ, Aladesanmi A. Antimicrobial activity of the leaf extract of Eugenia uniflora. Phytotherapy Research, 3(6): 258-259, 1989.

Almeida LMS, et al. Micropropagação e Aclimatização de Aegiphila verticillata Vell.: Uma Espécie Arbórea Ameaçada de Extinção. Revista Árvore, 39(2): 305314, 2015.
Almeida MZ. 2011. Plantas Medicinais. Universidade Federal da Bahia. 221p.

Amoroso MCM. Uso e diversidade de plantas medicinais em Santo Antônio do Leverger, MT, Brasil. Acta Botânica. 2 (16):189-203, 2002.

Antiqueira LM, Moro RS. 2020. Remanescentes de Cerrado no Parque Nacional dos Campos Gerais, PR, p. 52-58. In: Campos MA, Azevedo DJ (orgs). Biodiversidade Brasileira: Aspectos do Estado Atual 2. Jessica Aparecida Prandel. 60p.

Avidos MFD, Ferreira LT. Frutos dos Cerrados. Biotecnologia Ciência e Desenvolvimento. 3 (15): 36-41, 2000.

Balestra AC. 2015. Estudo da administração de dois extratos da planta Pyrostegia venusta no tratamento da asma em um modelo animal. Dissertação (Mestrado em Ciências da Saúde). Universidade de São Paulo. 72p.

Bolzani VS, Trevisan LMV, Young MCM. Triterpenes of Palicourea rigida $\mathrm{H}$. B. K. Revista Latinoamericana de Química. 23: 20-21, 1992.

Brasil. Departamento de Apoio Técnico e Educação Permanente. Comissão Assessora de Plantas Medicinais e Fitoterápicos. 2019. Plantas Medicinais e Fitoterápicos. CRF-SP (Conselho Regional de Farmácia do Estado de São Paulo). 86p. Disponível em: < https:// www.crfsp.org.br/images/cartilhas/PlantasMedicinais. pdf> Acesso em: 10/06/2020.

Brasil. Ministério da Saúde. 2006. A fitoterapia no SUS e o Programa de Pesquisa de Plantas Medicinais da Central de Medicamentos. Ministério da Saúde. 148 p. Disponível em:<https://bvsms.saude.gov.br/ bvs/publicacoes/fitoterapia_no_sus.pdf > Acesso em: 10/06/2020.

Fidalgo O, Bononi VLR. 1989. Técnicas de coleta, preservação e herborização de material botânico. Instituto de Botânica, São Paulo. 62p.

Filgueiras, T.S. et al. Caminhamento: um método expedito para levantamentos florísticos qualitativos. Cadernos de Geociências, 12: 39-43, 1994.

Guimarães EF, Monteiro D. Piperaceae na reserva biológica de Poço das Antas, Silva Jardim, Rio de Janeiro, Brasil. Rodriguésia, 57 (3): 567-587, 2006.

Joy PP. 1998. Medicinal plants. Agricultural University. 210p.

Leitão SG, Silva MH, Kaplan MAC, Delle MF. Constituents of some Aegiphila species and neutralization of Crotalus durissus terrificus venom by Aegiphila lhotzkyana. Fitoterapia, 67 (4): 377-379, 1996.

Lima LB, Silva JF, Pott A, Cardoso CL. 2011. Avaliação antioxidante $e$ antiacetilcolinesterase das folhas $e$ caules de Psychotria carthagenensis Jacq. (Rubiaceae). Dissertação (Mestrado em Ciências). Universidade de São Paulo. 149p. 
Lorenzi H, Bacher L, Lacerda M, Sartori S. 2006. Frutas brasileiras e exóticas cultivadas (de consumo in natura). Instituto Plantarum de Estudos da Flora. 640p.

Maack R. 1948. Notas preliminares sobre clima, solos e vegetação do estado do Paraná. Arquivos de Biologia e Tecnologia. 2ed. 225p.

Massuqueto LL, Pontes HS, Mochiutti NFB, Moreira JC, Vale TF, Foltran AC. 2019. Proposta de plano de uso público para a Fenda da Freira, atrativo natural do Parque Nacional dos Campos Gerais, (PR), p. 357-364. In: Anais do XXXV Congresso Brasileiro de Espeleologia. Disponível em: <http://www.cavernas. org.br/anais35cbe/35cbe_357-364.pdf $>$. Acesso em: 26/08/2020.

Melo MS, Moro RS, Guimarães GB. 2014. Os Campos Gerais do Paraná, p. 17-21. In: Melo MS, Moro RS, Guimarães GB. (orgs.). Patrimônio Natural dos Campos Gerais do Paraná. Mario Sérgio de Melo. 229p.

Melo MS, Lopes MC, Boska MA. 2005. Furna do Buraco do Padre, Formação Furnas, PR. Feições de erosão subterrânea em arenitos devonianos da Bacia do Paraná, p.48-55. In: Winge M, Schobbenhaus C, Berbert-Born M, Queiroz ET, Campos DA, Souza CRG, Fernandes ACS (orgs). Sítios Geológicos e Paleontológicos do Brasil. 515p.

Mendonça FAC, Silva KF, Santos KK, Ribeiro Júnior KA, Sant'ana AE. Activities of some Brazilian plants against larvae of the mosquito Aedes aegypti. Fitoterapia, 76 (7-8): 629-636, 2005.

Miranda N, Urban T. 1998. Engenhos e barbaquás. Posigraf, 120p.

Monteiro SC, Brandelli CLC. 2017. Farmacobotânica: Aspectos Teóricos e Aplicação. Artmed. 302p.

Moreira DL, et al. Estudos fitoquímico e farmacológico de Piper gaudichaudianum Kunth (Piperaceae). Revista Brasileira de Farmácia, 82 (1): 29-32,2001.

Nascimento GC, Menezes ACS, Lacerda EP. Estudo Fitoquímico e Avaliação da Atividade Antitumoral e Citotoxicidade in vitro de Erythroxylum deciduum (Erythroxylaceae). Revista Processos Químicos, 10: 4455, 2013.
Oliveira HWC. 2011. Cerrado e Plantas Medicinais: Algumas Reflexões sobre o Uso e Conservação. Trabalho de Conclusão de Curso (Licenciatura em Ciências Naturais). Universidade de Brasília, 25p.

Oliveira TG, et al. Micropropagação de Croton antisyphiliticus Mart. Revista Ciência Rural, 41 (10): 1712, 2011.

OMS (Organização Mundial da Saúde). 1998. Regulatory situation of herbal medicines: a worldwide review. Relatório Técnico. 45p. Disponível em: <https:// apps.who.int/iris/handle/10665/63801> Acesso em: 15/06/ 2020.

Pereira ZV, et al. Levantamento das Plantas Medicinais do Cerrado Sensu Stricto da Fazenda Paraíso Dourados, MS. Revista Brasileira de Biociências, 5 (1): 249-251, 2007.

Ramos EC, Monteiro LJ, Antiqueira LMOR. 2019. Plantas Medicinais dos Campos Gerais: patrimônio natural, histórico e cultural da região. 1 ed. Universidade Tecnológica Federal do Paraná. 86p.

Rocha MM, et al. Potencial Antioxidante de Erythroxylum deciduum A. St. Hill. In Vitro e In Vivo. Revista Perspectiva, 39 (147): 7-12, 2015.

Ritter LMO, Ribeiro MC, Moro RS. Composição florística e fitofisionomia de remanescentes disjuntos de Cerrado nos Campos Gerais. Revista Biota Neotropica, 10 (3): 379-414, 2010.

Theoduloz C, Franco L, Ferro EB, Schmeda-Hirchmann G. Xanthine oxidase inhibitory activity of Paraguayan Myrtaceae. Journal of Ethnopharmacology, 24: 179183, 1988.

Vieira MC. Nitrogênio e fósforo no desenvolvimento inicial da guavira Campomanesia adamantium (Cambess.) O. Berg cultivada em vasos. Revista Brasileira de Plantas Medicinais. 13: 542-545, 2011.

\section{Biodiversidade Brasileira - BioBrasil. Edição Temática: PIBIC n.1, 2022}

http:/www.icmbio.gov.br/revistaeletronica/index.php/BioBR

Biodiversidade Brasileira é uma publicação eletrônica científica do Instituto Chico Mendes de

Conservação da Biodiversidade (ICMBio) que tem como objetivo fomentar a discussão e a disseminação de experiências em conservação e manejo, com foco em unidades de conservação e espécies ameaçadas.

ISSN: 2236-2886 